\title{
Self-pulsation and excitability mechanism in silicon-on-insulator microrings
}

\author{
T. Van Vaerenbergh ${ }^{1,3}$, M. Fiers ${ }^{1,3}$, P. Mechet ${ }^{1,3}$, T. Spuesens ${ }^{1,3}$, R. Kumar $^{1,3}$, \\ G. Morthier ${ }^{1,3}$, K. Vandoorne ${ }^{1,3}$, B. Schneider ${ }^{1,3}$, B. Schrauwen ${ }^{2}$, J. Dambre ${ }^{2}$ and P. \\ Bienstman $^{1,3}$ \\ ${ }^{1}$ Photonics Research Group (INTEC), Ghent University - imec, \\ ${ }^{2}$ Electronics and Information Systems (ELIS), Ghent University, \\ ${ }^{3}$ Center for Nano- and Biophotonics (NB-Photonics), Ghent University, \\ Sint-Pietersnieuwstraat 41, B-9000 Ghent, Belgium
}

\begin{abstract}
In this paper, we demonstrate excitability in a silicon-on-insulator microring. Neglecting the fast light dynamics simplifies the calculation of the excitability onset. Excitable microrings will be useful as a basic building block in all-optical neural networks.

(C) 2012 Optical Society of America

OCIS codes: (190.4390) Nonlinear optics, integrated optics; (200.4700) Optical neural systems; (230.1150) Alloptical devices
\end{abstract}

\section{Introduction}

For a well-chosen wavelength and power setting of the input light, self-pulsation and/or excitability is observed in numerous passive microcavities, such as 2D Photonic Crystal (PhC) resonators [1], PhC nanocavities [2], Silicon-OnInsulator (SOI) microdisks [3] and SOI microrings [4, 5]. In all those cavities, a high $Q / V$-ratio enhances light-matter interaction. In Silicon-on-Insulator (SOI) cavities, Two Photon Absorption (TPA) generates both free carriers and heat. Other heating mechanisms are surface state absorption and Free Carrier Absorption (FCA). The presence of the free carriers induces a blue-shift of the resonance wavelength, known as Free Carrier Dispersion (FCD), while the heating of the cavity induces a red-shift due to the thermo-optic effect. The difference in timescale between the fast free carrier dynamics and the slow heating effects results in self-pulsation in whispering gallery mode cavities such as microdisks and microrings [3-6]. In InP-based 2D PhC resonators or PhC nanocavities a similar type of self-pulsation is visible [1,2], even though the main heating and free carrier generation mechanism in this material system is Single Photon Absorption (SPA) instead of TPA. Moreover, close to the self-pulsation region excitability is perceived both in In-P PhC cavities and SOI microrings $[1,2,5]$.

\section{Experimental set up and results}

We designed an all-pass ring with a $550 \mathrm{~nm} \times 220 \mathrm{~nm}$ cross section, a $4.5 \mu \mathrm{m}$ radius, a $250 \mathrm{~nm}$ gap, with a bus waveguide bended with the same curvature as the ring. This ring has a resonance at $1530.708 \mathrm{~nm}$ with $\delta \lambda_{3 d B} \approx 20 \mathrm{pm}(\mathrm{with}$ resonance splitting $\delta \lambda_{p s} \approx 20 \mathrm{pm}$ ) and extinction ratio $8 \mathrm{dBm}$. A temperature controller guarantees that chip temperature deviations are below $\pm 0.03 \mathrm{~K}$. Light of a tunable laser (TL) is coupled in and out the chip by grating couplers. The ring output is measured with a $10 \mathrm{GHz}$ photodiode and visualized with a $1 \mathrm{GHz}$ real-time scope. When this microring is pumped with a CW input signal, with well-chosen input wavelength and power, sufficiently strong perturbations can trigger excitations (Fig. 1).

\section{Theoretical analysis}

This dynamic behaviour can be described accurately using temporal coupled mode theory [3]. The light that circulates in the cavity can be represented by a complex amplitude. The temperature difference of the cavity with the surroundings $\Delta T$ and the amount of free carriers $N$ count as two additional dynamic variables. This model allows to calculate realistic steady-state curves. From linear stability analysis the oscillation onset can be identified as an Andronov-Hopf bifurcation [3,5]. Moreover, phase-plane analysis illustrates that the self-pulsation is caused by the interplay between temperature and free carrier effects, indicating that the fast light dynamics (i.e. the cavity build-up time) are less relevant [1,5]. However, a thorough analysis of the influence of the different material parameters on the oscillation and excitability onset is still missing, as it is not straightforward to calculate bifurcation diagrams of this 4D-system. 


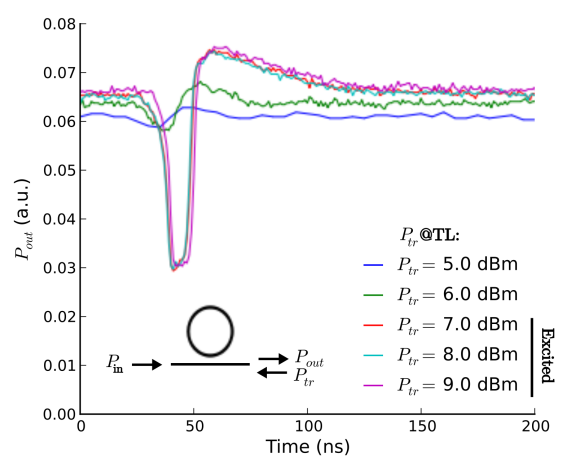

Fig. 1. If the trigger power $P_{t r}$ is sufficiently high ( $\geq 7 \mathrm{dBm} @ \mathrm{TL}$ ) the ring excites with a fixed pulse shape, while for lower trigger powers subthreshold oscillations are visible. The $4 \mathrm{dBm}$ pump light is detuned at $\delta \lambda=-4 \mathrm{pm}$ from the $\lambda_{r}=1530.708 \mathrm{~nm}$ resonance. The trigger light is tuned $\delta \lambda_{t r}=9 \mathrm{pm}$ near another ring resonance at $\lambda_{r^{\prime}}=1550.671 \mathrm{~nm}$. Power values are those at the output of the lasers. Due to the grating coupler the on-chip input power of the ring is expected to be $\sim 6 \mathrm{dBm}$ lower.

In this paper, we eliminate the fast light dynamics in the equations of motion and rescale $\Delta T$ and $N$ to resp. $\Theta=$ $\Delta T \frac{2 Q \frac{d n}{d T}}{n_{g}}$ and $n=N \frac{2 Q\left|\frac{d n}{d N}\right|}{n_{g}}$. As $\delta=\left(\omega-\omega_{r}\right) \tau_{p h}$ is the detuning of the input light to the resonance frequency $\omega_{r}$ of a cavity with photon lifetime $\tau_{p h}$ (and thus $Q=\omega_{r} \tau_{p h} / 2$ ), $\Theta$ and $n$ can be interpreted as the absolute value of the induced detuning shift caused by resp. $\Delta T$ and $N$. We then end up with a dimensionless $2 \mathrm{D}$-system:

$$
\begin{gathered}
\frac{d \Theta}{d t}=-\Theta+\frac{p(1+e f n)}{(1+f n)^{2}+(\delta+\Theta-n)^{2}}, \\
\frac{d n}{d t}=\frac{1}{\varepsilon}\left[-n+\left(\frac{p q}{(1+f n)^{2}+(\delta+\Theta-n)^{2}}\right)^{2}\right] .
\end{gathered}
$$

For the power and energy normalisation we used the thermal and electric characteristic intrinsic power of the cavity and $P_{0}^{t h}=\frac{\rho_{S i} c_{p, S i}}{4 \frac{d n}{d_{g} T} \tau_{t h} \eta_{l i n} \Gamma_{t h}} \frac{V_{t h}}{Q_{i}}\left(\frac{1+k}{k}\right)^{2}$ and $P_{0}^{e l}=\sqrt{\frac{\hbar \omega_{r}^{3}}{4 \frac{d n}{d n} \mid} \tau_{f c} \Gamma_{F C A} \beta_{S i} \nu_{g}^{2}} \frac{V_{F C A}}{Q_{i}^{3 / 2}}\left(\frac{1+k}{k}\right)^{3 / 2}$. Here, $\tau_{t h}$ and $\tau_{f c}$ are the relaxation times for resp. the temperature and the free carriers; $\beta_{S i}$ is the constant governing TPA; $c_{p, S i}$ the thermal capacity; $\rho_{S i}$ the density of silicon and $n_{g}$ is the group index. We also use the effective volumes $V_{\alpha}$ and confinements $\Gamma_{\alpha}$ corresponding with a physical effect $\alpha$ defined in [3,7]. $\eta_{\text {lin }}$ is the fraction of the linear loss that is absorbed $\left(\approx 0.4\right.$ in SOI [6,7]). $Q_{i}$ is the intrinsic Q-factor of this cavity. Similar to [7], $k=\frac{\tau_{c}}{\tau_{l}}$ is the ratio of good loading (lifetime $\tau_{c}$ ) to the parasitic and intrinsic loss channels (lifetime $\tau_{l}$ ) of the resonator. The loaded Q-factor of the cavity is then $Q=Q_{i} k /(1+k)$. For a critically coupled cavity $k=1$.

$p=P_{\text {in }} /\left(P_{0}^{t h} \frac{1+k}{2}\right)$ is the normalized version of the input power and $q=\frac{P_{0}^{t h}}{P_{0}^{e l}}$; determines the relative importance of the thermo-optic effect versus FCD. $f=\alpha_{H}^{-1}$ compares the broadening of the resonance width due to FCA with FCD, with $\alpha_{H}$ the Henry factor [2]. $e=\frac{1+k}{k \eta_{l i n}}$ and $e f$ represents the additional heating due to FCA. $\varepsilon=\tau_{f c} / \tau_{t h}$, while time is expressed in units $\tau_{t h}$.

Eq. 1-2 still exhibit excitability (Fig. 2(a), simulated with Caphe [8]), while the dimensionality reduction allows a semi-analytic calculation of both the bistability (BI) onset and the Hopf bifurcation, i.e., the self-pulsation (SP) onset (Fig. 2(b)). A fold bifurcation of limit cycles (LC) curve starts from a Generalized Hopf (GH) bifurcation on the Hopf bifurcation curve. In the thin region between the LC fold and Hopf bifurcation curves a stable fixed point (FP) co-exists with a stable LC. Thereby, for $\delta>\delta_{G H}$ and $p$ sufficiently strong, but still smaller than the LC fold, the microring is excitable. The LC fold is calculated using PyDSTool [9]. 

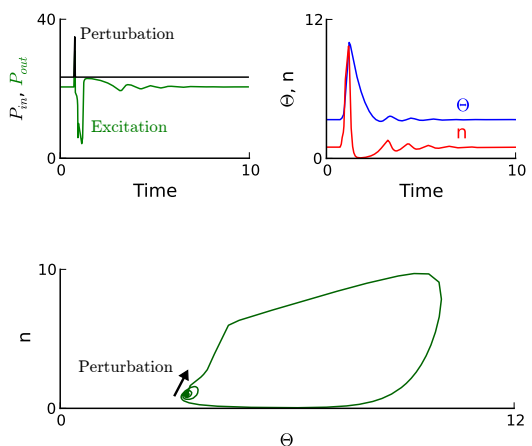

(a) Example timetrace of excitability at $\delta=0.5$

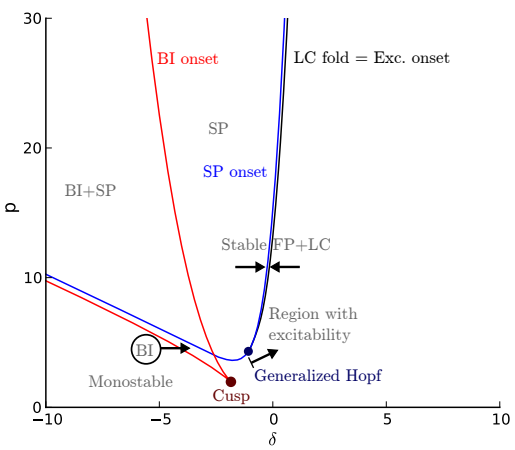

(b) Bifurcation plane: power $p$ vs detuning $\delta$.

Fig. 2. Eq. 1-2 can be used to simulate and analyse the excitability mechanism in a microring. We illustrate our approach for a typical critically coupled all-pass SOI microring with self-pulsing behaviour $(q=0.397, \varepsilon=0.0815, f=0.0714, e=5)$.

\section{Conclusion}

Excitability in a silicon-on-insulator all-pass microring is experimentally observed. If the fast light dynamics are neglected, both the self-pulsation and excitability onsets can be analytically calculated. Thereby, it is possible to predict the size of the excitability region for a given microring. This analysis can be used in the design of microring based all-optical neural networks.

Acknowledgments

This work is supported by the interuniversity attraction pole (IAP) Photonics@ be of the Belgian Science Policy Office and the ERC NaResCo Starting grant. T. Van Vaerenbergh is supported by the Flemish Research Foundation (FWOVlaanderen) for a PhD Grant. M. Fiers acknowledges the Special Research Fund of Ghent University.

\section{References}

1. A. Yacomotti, et al., "Fast Thermo-Optical Excitability in a Two-Dimensional Photonic Crystal," Physical Review Letters 97, 6-9 (2006).

2. M. Brunstein, et al., "Excitability and self-pulsing in a photonic crystal nanocavity," Physical Review A 85, 1-5 (2012).

3. T. J. Johnson, et al., "Self-induced optical modulation of the transmission through a high-Q silicon microdisk resonator." Optics Express 14, 817-31 (2006).

4. W. H. P. Pernice, et al., "Time-domain measurement of optical transport in silicon micro-ring resonators." Optics Express 18, 18,438-52 (2010).

5. T. V. Vaerenbergh, et al., "Cascadable Excitability in microrings," Optics Express (2012).

6. G. Priem, et al., "Optical bistability and pulsating behaviour in Silicon-On-Insulator ring resonator structures." Optics Express 13, 9623-8 (2005).

7. P. Barclay, et al., "Nonlinear response of silicon photonic crystal microresonators excited via an integrated waveguide and fiber taper." Optics express 13, 801-20 (2005).

8. M. Fiers, et al., "Time-domain and frequency-domain modeling of nonlinear optical components on circuit-level using a node-based approach," Journal of the Optical Society of America B (2011).

9. R.H. Clewley, et al., "PyDSTool, a software environment for dynamical systems modeling." (2007). 